International Journal of Engineering \& Technology, $7(4.10)(2018)$ 1070-1074
International Journal of Engineering \& Technology
SPC
Website: www.sciencepubco.com/index.php/IJET
Research paper

\title{
Combined Effects of Inclined Lorentzian forces and Melting heat transfer on Radiating Flow of Carbon Nanofluid past a Stretching Cylinder
}

\author{
Sreenivasulu ${ }^{1}$ P. and Poornima ${ }^{2 *}$ T. \\ ${ }^{1}$ Department of Mathematics, Sri Venkateswara College of Engineering for Women, Tirupati-517501,India. \\ ${ }^{2}$ School of Advanced Sciences, VIT University, Vellore-632014, India. \\ *Corresponding author E-mail: poornima.t@vit.ac.in
}

\begin{abstract}
An analysis was made to study the effects of non-uniform heat source or sink and aligned magnetic field effect on boundary layer flow of carbon nanofluid past a stretching cylinder with melting heat transfer and radiation. The Prandtl boundary layer equations are transformed into highly nonlinear ordinary differential equations utilizing similarity variables. The final resolved system is explained with shooting method. The upshots of governing factors on the velocity, temperature, surface skin friction and rate of heat transfer are discoursed with the help of graphs. MWCNT performance is better than SWCNT in case of fluid flow. SWCNTs transfers more heat from the fluid rather than multi wall tubes.Comparison of our numerical results with available literature works and shown a good agreement.
\end{abstract}

Keywords: Convective slip ; Dissipation; Exponential stretching sheet; Hydrodynamics; Lorentzian force; Melting heat transfer; Radiation.

\section{Motivation}

Electrically magnetic field applied transversely normal to the fluid flow aligned is studied under Maxwell's law. This part has wide applications in the power generators, magnetic shielding, bearings, and electrically leading fluid. Sharma and Singh [1] analyzed the heat sink/source phenomena with varying thermal conductivity on magnetohydrodynamic pin point flow. Aligned magnetic effects on plasma structures were explained by Ergun et al. [2]. Sulochana et al. [3] investigated the inclined Lorenztian forces and Soret and Dufour effects. At high temperatures, radiation effect is not negligible. As it has many utilizations in designing of equipment which has capable of bearing high temperature. Impact of thermal heat transfer in stagnated flow of carbon water nanofluid was studied by Taswar et al.[4]. Rashid et al. [5] explained the radiation effects on water based metallic nanoparticles with prescribed surface temperature. Mahapatra and Gupta [6] inspected the stagnated flow with heat transfer impact along the elongated sheet. Pop et al. [7] explored the influence of radiative transfer of heat on the flow along stretched sheet.

Today's requirement is compact heat transfer systems. In developing such type of efficient system, lies the true work of researchers. Such type of work is useful in developing thermal engineering. In order to enhance the thermal conductivity in heat transferring, Choi [8-9] introduced nanoparticles which when combined with poor conductive base fluids to form a colloid, called nanofluids [10]. They are of man-made fluids whose particles may be of spherical or cylindrical shapes called tubes [11]. It is proved experimentally those tubes ahead spherical and other shapes of parti cles. CNT's are proven as wonder technology as it combines with kerosene or engine oil shows excellent thermal transfer. CNT are enclosed by graphene layers. Basing on the layers CNT are of two types SWCNT and MWCNT (Xue [12]). They are used in Magnetic shielding, batteries, waste recycling, transistors, solar storage, thermal conductors, biosensors, etc. And also used in medical and social purposes such as water purification process [13]. Analysis of flow of temperature on MHD carbon nanotube past a wedge, explained by Khan et al. [14]. Three dimensional rotating flow with CNT embedded in a porous medium was investigated by Hayat et al. [15].

In the recent era, in many advanced technological processes, melting /freezing of any object plays an important role. Current researchers showing much interest in discovering heat transfer due to melting because of its enormous applications in thawing and solidification procedures such as welding process, moulding and industrial manufacturing process, lava/magma solidification, heat transportation melting of permafrost, the freezing treatment of sewage, softening of frozen grounds. Effect of induced magnetic field with melting heat transfer past a stretching sheet was examined by Gireesha et al. [16]. Taswar Hayat et al.[17] studied carbon nanotubes with melting heat transfer condition on variable thickness surface. Adegbie et al. [18] described the melting heat transfer boundary condition on micro polar fluids.

With the above awareness, an attempt is made to study the radiation and aligned magnetic field effects on boundary layer flow of two different types of CNTS namely SWCNT and MWCNT past a stretching sheet with melting heat transfer boundary condition.

\section{Numerical analysis}

Consider a steady 2D, incompressible laminar carbon water nanofluid past a stretching cylinder with partial slip. As the geom- 
etry is cylinder, coordinates are axially $x$ and radially $r$. We took SWCNT and MWCNT and applied an inclined Lorentzian force normal to the flow. Two equally strengthened force but in contradictory path creates a stretching velocity (Figure 1).

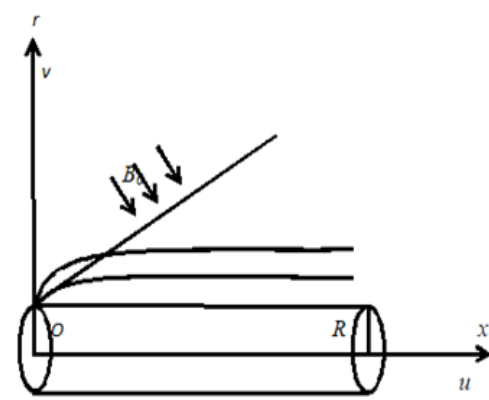

Fig.1: Physical model of the problem

Assumptions of Boussinesq's are taken into account and the mathematical model for the present discussion is posed here.

$$
\begin{aligned}
& \frac{\partial(r u)}{\partial x}+\frac{\partial(r v)}{\partial r}=0 \\
& u \frac{\partial u}{\partial x}+v \frac{\partial u}{\partial r}=U_{e} \frac{d U_{e}}{d x}+v_{n f}\left(\frac{\partial^{2} u}{\partial r^{2}}+\frac{1}{r} \frac{\partial u}{\partial r}\right)-\frac{\sigma B_{0}^{2}}{\rho_{n f}} \operatorname{Sin}^{2} \xi u \\
& u \frac{\partial T}{\partial x}+\frac{\partial T}{\partial r}=\alpha_{n f f}\left(\frac{\partial^{2} T}{\partial r^{2}}+\frac{1}{r} \frac{\partial T}{\partial r}\right)+\frac{1}{\left(\rho c_{p}\right)_{n f}} \frac{16 \sigma^{*} T_{s}^{3}}{3 k^{*}}\left(\frac{\partial^{2} T}{\partial r^{2}}+\frac{1}{r} \frac{\partial T}{\partial r}\right)+\frac{1}{\left(\rho c_{p}\right)_{n f}} q^{\prime \prime}
\end{aligned}
$$

The boundary conditions for the velocity and temperature field are

$$
\begin{gathered}
u=u_{w}(\mathrm{x})+\delta \frac{\partial u}{\partial r}, \quad v=0, \quad T=T_{m} \quad \text { at } r=R d \\
u \rightarrow u_{e}(\mathrm{x})=\frac{U_{x} x}{l}, \quad T \rightarrow T_{\infty} \quad \text { as } \quad r \rightarrow \infty \\
k_{n f}\left(\frac{\partial T}{\partial r}\right)_{r=R}=\rho_{n f}\left[\lambda_{1}+c_{s}\left(T_{m}-T_{0}\right)\right] v(R d, x)
\end{gathered}
$$

where $u, v$ are radial and axial velocity components in $x, r$ directions respectively. $\xi$ is the aligned angle, $\sigma$ is the electric conductivity, $B_{0}$ is the magnetic field strength, $T$ is the temperature of the fluid, $T_{m}$ is the temperature of the melting surface, $T_{\infty}$ is the quiescent flow temperature, $\rho, \mu, v, C p$, and $k$ are respectively the density, dynamic viscosity, kinematic viscosity, specific het at constant pressure and thermal conductivity, $k^{*}$ is the mean absorption coefficient, $\sigma^{*}$ is the Stefan-Boltzmann Constant, $u_{w}$ is the stretching velocity, $u_{e}$ is the free stream velocity, $c_{s}$ is the surface heat capacity, $R d$ is the radius of the cylinder, $l$ is the characteristic length, $\lambda_{1}$ is the fluid latent heat.

The condition represented in equation (5) is the melting heat transfer condition. The physical concept is that surface heat conduction is the ratio of melting heat to the heat required to improve $T_{0}$ to $T_{m}$. The variable thermal sink/generation $q^{\prime \prime \prime}$ has the form

$q^{\prime \prime \prime}=\frac{u_{w}}{x} \frac{k_{f}}{v_{f}}\left(B\left(T-T_{\infty}\right)+A f^{\prime}(\eta)\left(T_{w}-T_{\infty}\right)\right)$

where A,B are the heat sink/source parameters, $v_{f}$ is the base fluid kinematic viscosity, $k_{f}$ is the base fluid thermal conductivity.

CNTs are elliptical tubes with large axial ratio. Maxwell theory with these types of CNTs and its space distributions was proposed by Xue [12]

$$
\begin{aligned}
& \rho_{n f}=(1-\phi) \rho_{f}+\phi \rho_{C N T}, \alpha_{n f}=\frac{k_{n f}}{(\rho C p)_{n f}},\left(\rho C_{p}\right)_{n f}=(1-\phi)\left(\rho C_{p}\right)_{f}+\phi\left(\rho C_{p}\right)_{C N T}, \\
& (\rho \beta)_{n f}=(1-\phi)(\rho \beta)_{f}+\phi(\rho \beta)_{C N T}, \mu_{n f}=\frac{\mu_{f}}{(1-\phi)^{2.5}}, \frac{k_{n f}}{k_{f}}=\frac{1-\phi+2 \phi \frac{k_{C N T}}{k_{C N T}-k_{f}} \ln \frac{k_{C N T}+k_{f}}{2 k_{f}}}{1-\phi+2 \phi \frac{k_{f}}{k_{C N T}-k_{f}} \ln \frac{k_{C N T}+k_{f}}{2 k_{f}}} .
\end{aligned}
$$

where $\phi$ is the nanotube volume fraction, the subscripts $n f, f$ and $C N T$ are respectively represents the nanofluid, base fluid and Carbon nanotubes

Similarity variables are applied to equations (1) - (3) and the boundary conditions (4) - (5), in order to convert dimensional quantities to dimensionless. The below were the quantities utilized.

$$
\begin{aligned}
& \eta=\sqrt{\frac{U_{0}}{v_{f} l}}\left(\frac{r^{2}-R d^{2}}{2 R d}\right), \psi=\sqrt{u_{w} v_{f}} x R d f(\eta), u=\frac{U_{0} x}{l} f^{\prime}(\eta), v=-\sqrt{\frac{U_{0} v_{f}}{l}} \frac{R d}{r} f(\eta), \\
& \theta=\frac{T-T_{m}}{T_{\infty}-T_{m}}, M=\frac{\sigma B_{0}^{2}}{\rho_{f} l}, \lambda=\frac{U_{\infty}}{U_{0}}, \gamma=\frac{1}{R d} \sqrt{\frac{V_{f} l}{U_{0}}}, \operatorname{Pr}=\frac{\mu_{f}(C p)_{f}}{k_{f}}, R=\frac{4 \sigma^{*} T_{\infty}^{3}}{k^{*} k_{f}}, \\
& \alpha=\sqrt{\frac{\mu_{f} \rho_{f} U_{0}}{l}} \delta, \beta=\frac{(C p)_{f}\left(T_{\infty}-T_{m}\right)}{\lambda_{1}+c_{s}\left(T_{m}-T_{0}\right)}, u_{w}(x)=\frac{U_{0} x}{l}, u_{e}(x)=\frac{U_{\infty} x}{l}, \operatorname{Re}_{x}=\frac{u_{w} x}{v_{f}}
\end{aligned}
$$

In view of equation (8), (2) and (3) turns to the ODE form.

$$
\begin{aligned}
& (1+2 \gamma \eta) f^{\prime \prime \prime}+2 \gamma f^{\prime \prime}+(1-\phi)^{2.5}\left(f f^{\prime \prime}-f^{\prime 2}+\lambda^{2}\right)-(1-\phi)^{2.5} M \operatorname{Sin}^{2} \xi f=0 \\
& \left(\frac{k_{n f}}{k_{f}}+\frac{4}{3} R\right)\left((1+2 \gamma \eta) \theta^{\prime \prime}+2 \gamma \theta^{\prime}\right)+\operatorname{Pr}\left(1-\phi+\phi \frac{(\rho C p)_{C N T}}{(\rho C p)_{f}}\right) f \theta^{\prime}+A f^{\prime}+B \theta=0
\end{aligned}
$$

Subject to the boundary conditions

$$
\begin{aligned}
& f^{\prime}(0)=1+\alpha f^{\prime \prime}(0), \quad f(0)=0, \quad \theta(0)=0 \\
& f^{\prime}(\infty)=A, \quad \theta(\infty)=1 \\
& \operatorname{Pr}\left(1-\phi+\phi \frac{\rho_{C N T}}{\rho_{f}}\right) f(0)+\beta \frac{k_{n f}}{k_{f}} \theta^{\prime}(0)=0
\end{aligned}
$$

where $M$ is the magnetic field, $R$ is the radiation parameter, $P r$ is the Prandtl number, $A$ is the stretching ratio parameter, $\gamma$ is the curvature of the cylinder, $\alpha$ is the velocity slip parameter, $\beta$ is the Stefan numbers combining $c_{p}\left(T_{\infty}-T_{m}\right) / \lambda_{1}$ and $c_{s}\left(T_{m}-T_{0}\right) / \lambda_{1}$ of the liquid and solid phases.

Engineering quantities of our interest are the $C_{f}$ and $N u_{x}$ which are defined as

$$
\sqrt{\operatorname{Re}_{x}} C_{f}=\frac{1}{(1-\phi)^{2.5}} f^{\prime \prime}(0), \quad \frac{N u_{x}}{\sqrt{\operatorname{Re}_{x}}}=-\left(\frac{k_{n f}}{k_{f}}+\frac{4}{3} R\right) \theta^{\prime}(0)
$$

where $R e_{x}$ is the Reynolds number,

Table 1: Base fluid and particles thermos-physical properties

\begin{tabular}{cccc}
\hline Physical & Basefluid & \multicolumn{2}{c}{ CNTs(particles) } \\
\cline { 2 - 4 } properties & Water & SWCNT & MWCNT \\
\hline $\begin{array}{c}\text { Density }\left(\rho-\mathrm{kg} / \mathrm{m}^{3}\right) \\
\text { Specific heat capaci- } \\
\text { ty }\left(C_{p}-\mathrm{J} / \mathrm{kg} \mathrm{K}\right)\end{array}$ & 997 & 2600 & 1600 \\
$\begin{array}{c}\text { Thermal conductivi- } \\
\text { ty }(k-\mathrm{W} / \mathrm{m} \mathrm{K})\end{array}$ & 0.613 & 425 & 796 \\
\hline
\end{tabular}

\section{Results and discussion}

The highly coupled non-linear equations shown in equations (9) and (10) with respect to the conditions (11) and (12) is solved numerically by using shooting method. The resultant initial problem is solved by employing fourth order Runge-Kutta technique. Numerical values are computed using Mathematica software for a set of values of physical parameters. The effects are portrayed graphically in Figs. $2-11$ and discussed elaborately.

We found an excellent agreement with the existing literature for some reduced cases that of Sharma and Sing[1], Mahapatra and Gupta[6] and Pop et al. [7]. 


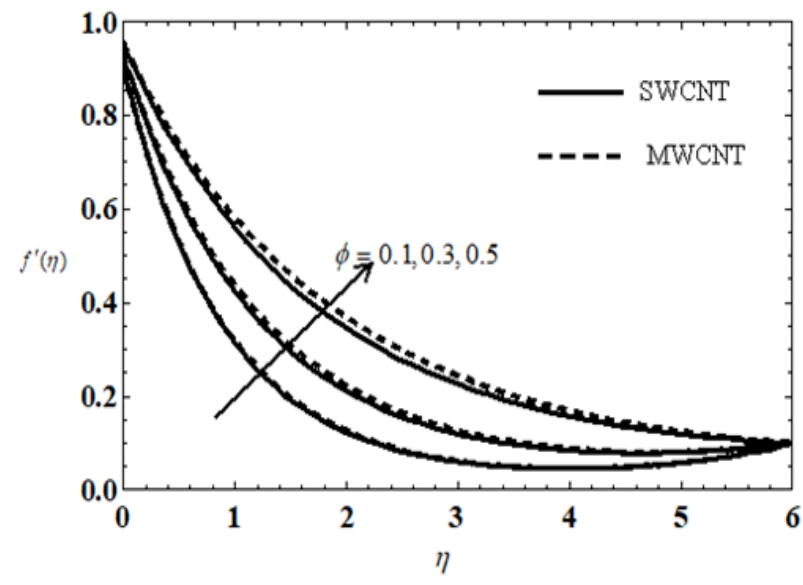

Fig.2: Velocity profiles for different $\phi$

The influence of nanoparticle volume fraction on velocity and temperature are respectively shown in Figs. 2 and 3. Nanoflow velocity escalates on improving the size of the nanotubes. We know the fact that increase in the boundary layer thickness increases the movement of the fluid flow and the heat of the fluid drops for escalating volume fraction.

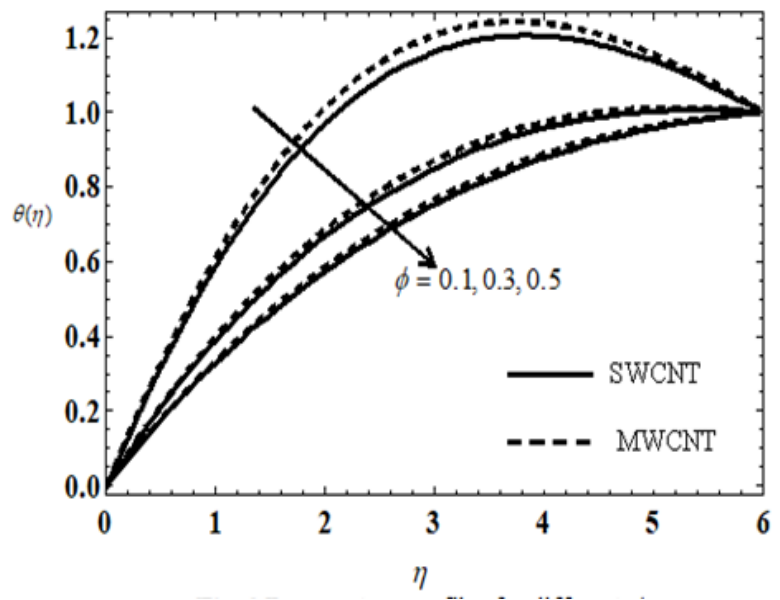

Fig.3: Temperature profiles for different $\phi$

Table 2: Comparison of results for $-f^{\prime \prime}(0)$ when $A=B=M=\alpha=\beta=\gamma=$ 0 with present study.

\begin{tabular}{|c|c|c|c|c|}
\hline$A$ & $\begin{array}{c}\text { Sharma and } \\
\text { Singh [1] }\end{array}$ & $\begin{array}{c}\text { Mahapatra } \\
\text { and Gupta [6] }\end{array}$ & $\begin{array}{c}\text { Pop } \text { et al. } \\
\text { [7] }\end{array}$ & Present Study \\
\hline 0.1 & -0.9694 & -0.9694 & -0.9694 & -0.969388 \\
\hline 0.2 & -0.9181 & -0.9181 & -0.9181 & -0.918092 \\
\hline 0.5 & -0.6673 & -0.6673 & -0.6673 & -0.667302 \\
\hline 0.7 & & & & -0.433427 \\
\hline 0.8 & & & & -0.299279 \\
\hline 0.9 & & & & -0.154552 \\
\hline 1.0 & & & & 0.000000 \\
\hline
\end{tabular}

We see from the graph in the nanoflow motion, MWCNT dominates SWCNT while the reverse is happening for temperature. Fig. 4 represents the velocity profiles for different values of aligned magnetic field angle. From this figure, it is clear that the nanoflow velocity descends for ascending Lorentzian force.

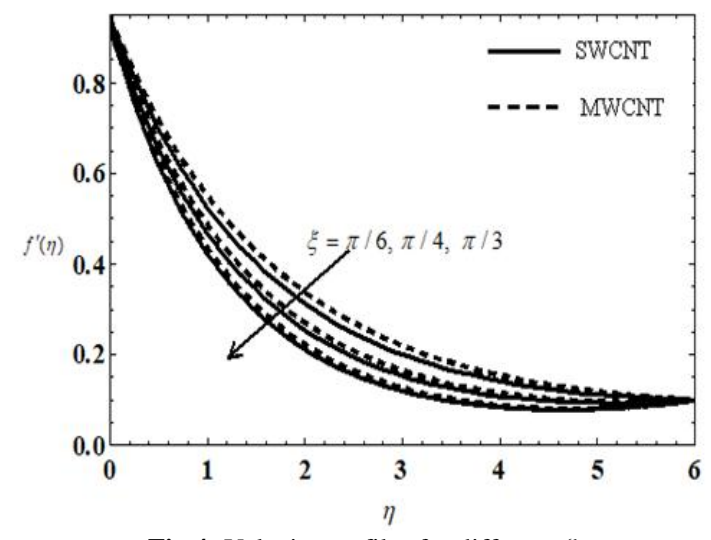

Fig.4: Velocity profiles for different $\xi$

The impact of $\gamma$ on the momentum and temperature are illustrated in Figs. 5 and 6. We see that the fluid motion drops near the surface and after $\eta=0.5$, a reverse trend is observed for escalating curvature parameter (Fig. 5). Fig.6 shows the nanoflow warmth rises initially heat and contradicting behavior is observed after the inflection point $\eta=4.5$. Also it is observed that in both the cases the MWCNT is slightly higher than the SWCNT. The fact that the conduction processes is more prominent near the surface due to decreases the radius of the cylinder for higher values of $\gamma$.

Fig.7 shows clearly that the fluid velocity drops for an increase in $\alpha$ and this is of course the force enforced on the nanoflow by the stretching surface. One can observe that Multi wall CNTs have higher values compared to Single walled tubes.

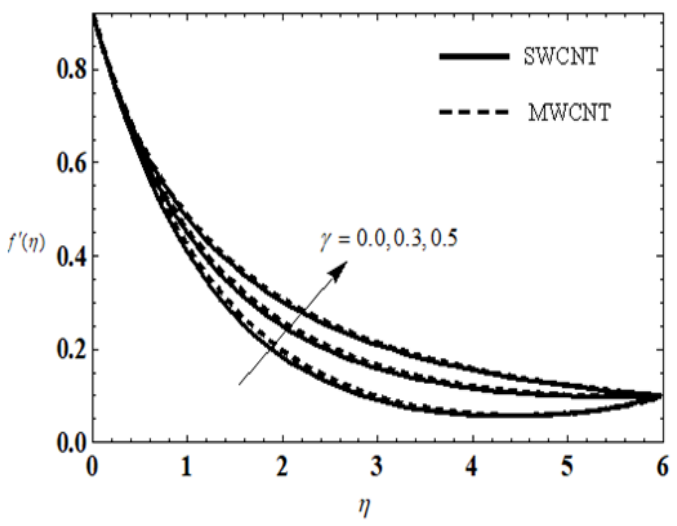

Fig.5: Velocity profiles for different $\gamma$

Increasing $\beta$ value enhances the warmth in the fluid because for more heat from the hot fluid get transferred to melting surface (Fig. 8). Velocity profiles uplifts with an increasing $\beta$ values (Fig. 9). One can notice that MWCNT dominates the flow whereas SWCNTs transfers heat more than MWCNTs. Here $\beta=0$ implies absence of melting mode heat transfer.

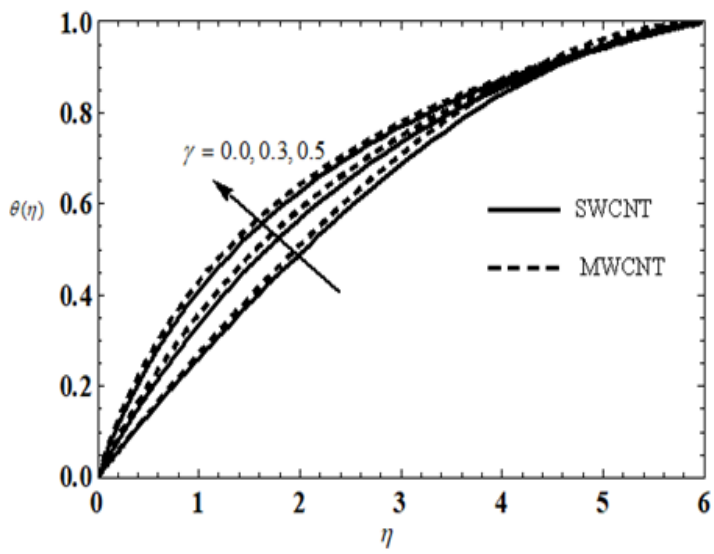

Fig.6: Temperature profiles for different $\gamma$ 


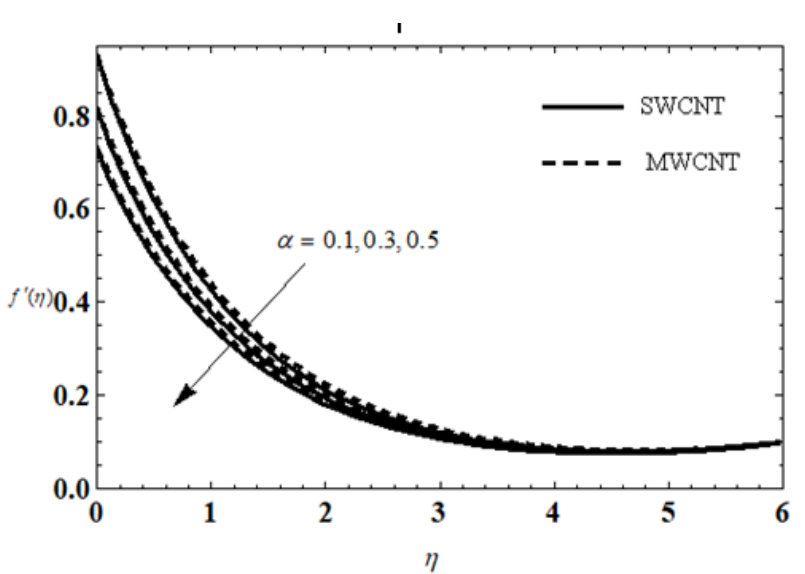

Fig.7: Velocity profiles for different $\alpha$

The influence of heat source or sink parameters A and B on the temperature are shown in figs. 10 and 11. From Fig. 10, it is pointed out that the nanoflow temperature reduces with rising $A$ and $B$. It is fact that the heat generates for the case $\mathrm{A}>0$ and $\mathrm{B}>$ 0 whereas warmth loses from the sheet for $\mathrm{A}<0$ and $\mathrm{B}<0$.

Also it is observe that the temperature of the fluid is higher in case of MWCNT than that of SWCNT.

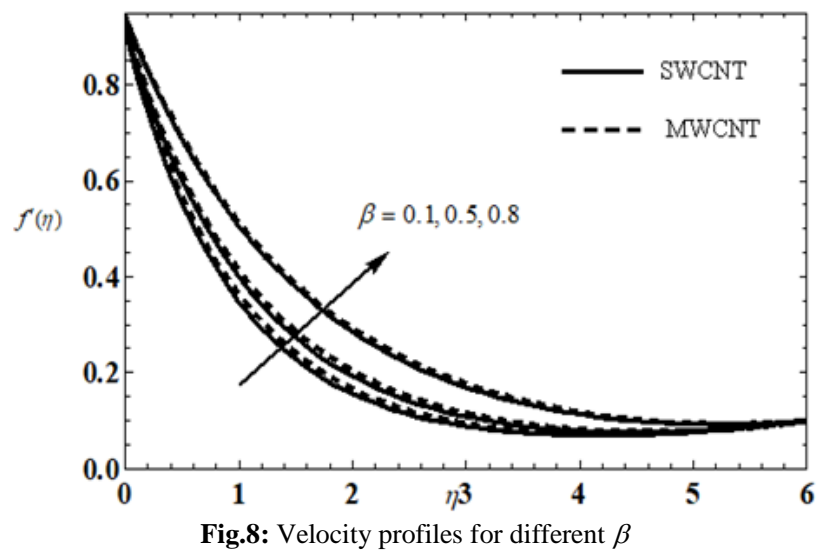

Table 3 represents a set of tabulated values for surface skin friction and the rate of heat transfer. It is noticed that surface shear stress descends while the $N u_{x}$ enhances for improving nanotubes size. The friction factor near the stretching surface is more when the radius of the cylinder increases.

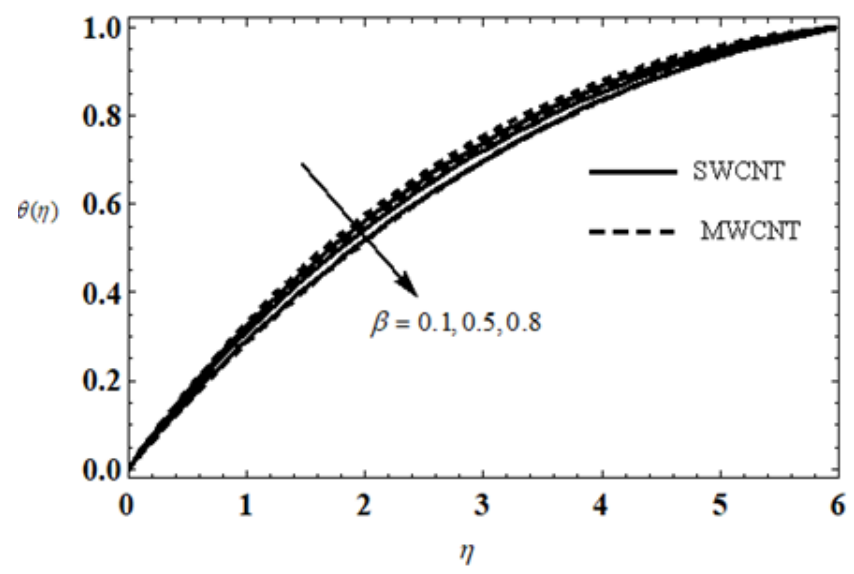

Fig.9: Temperature profiles for different $\beta$

Rate of heat transfer reduces for increase $\gamma$ values. The surface skin decreases and heat transfer rate increases with an increase in stretching ratio parameter. Also it is evident that for higher values of aligned magnetic field angle, decreases the surface skin friction and heat transfer rate. The raising values of radiation parameter results to increase in both the surface skin friction and rate of heat transfer.

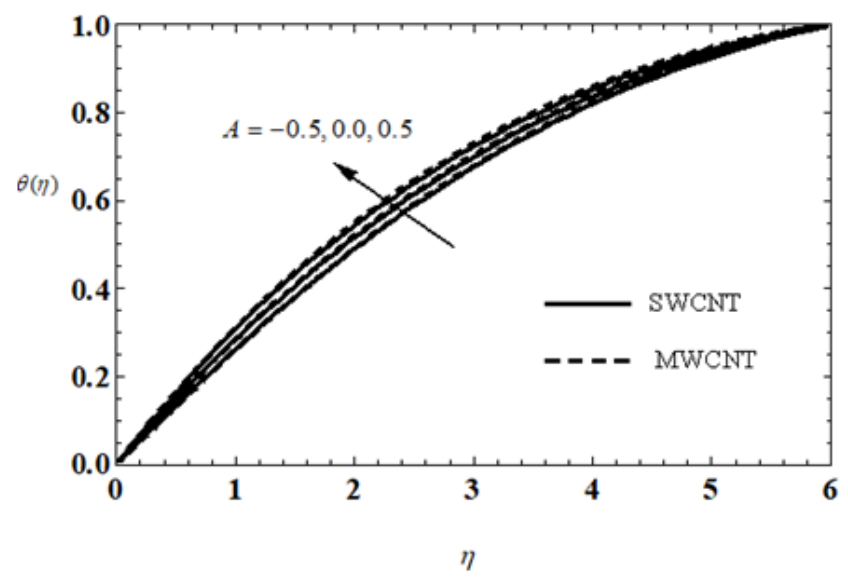

Fig.10: Temperature profiles for different $A$

Ascending slip factor reduces the friction coefficient while the convective heat transfer rate enhances. The surface skin friction increases and heat transfer rate decreases with an increase in melting heat transfer parameter increases. Skin friction as well as heat transfer rates are decreases with an increase in non-uniform heat source or sink parameters.

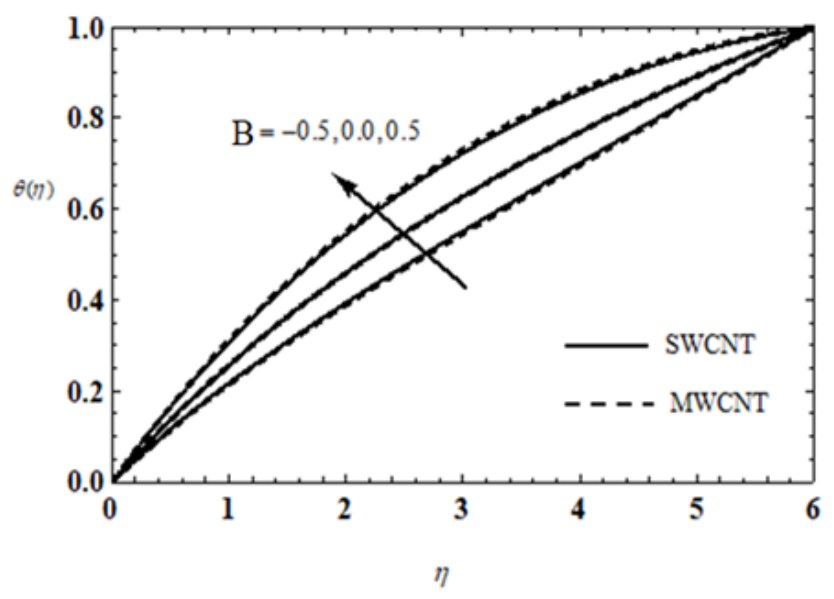

Fig.11: Temperature profiles for different $B$

\section{Conclusion}

An analysis is made to study the upshot of aligned magnetic field and melting heat transfer on CNTs imbedded radiated nanoflow past a stretching cylinder with non-uniform sink/generation. From the above investigation, one may conclude that

$>$ CNTs improve transfer of heat from the flow.

$>$ Nanoflow momentum profiles swiftly rises with an increase in nanoparticle volume fraction, curvature parameter and heat transfer parameter while it drops with an increase in inclined angle and slip parameter.

$>$ The temperature of the nanofluid rises with an increase in $\gamma$ or $R$ or $A^{*}$ and $B^{*}$ but it decreases with an increase in $\phi$ or $\xi$ or $\alpha$ or $\beta$.

$>$ Skin friction can be reduced for higher values of $\phi$ or $\lambda$ or $\xi$ or $\alpha$ or $\beta$ or $A^{*}$ or $B^{*}$ and it rises with an increase in $\gamma$ or $R$.

$>$ The rate of heat transfer increases with an increase in $\phi$ or $\lambda$ or $\xi$ or $\alpha$ or $\beta$ or $R$ and it shows an opposite behavior when rise in $\gamma$ or $A^{*}$ or $B^{*}$. 


\section{References}

[1] Sharma PR \& Singh G (2009), Effects of variable thermal conductivity and heat source or sink on MHD flow near a stagnation point on s linear stretching sheet. Journal of Applied Fluid Mechanics 2 , $1-21$.

[2] Ergun RE, Carlson CW, McFadden JP, Mozer FS, Muschietti L, Roth I \& Strangeway RJ (1998), Debye-Scale plasma structures associated with magnetic field aligned electric fields. Physics Review Letters 81, 826.

[3] Sulochana C, Sandeep N, Sugunamma V \& Rushi Kumar B (2016), Aligned magnetic field and cross-diffusion effects of a nanofluid over an exponentially stretching surface in porous medium. Applied Nanoscience 6,737-746.

[8] Choi SUS (1995), "Enhancing thermal conductivity of fluids with nanoparticles, in developments and applications of Non-Newtonian flows. ASME FED 231/MD 66, 99-103.

[9] Seok Pil Jang \& Choi SUS (2007),Effects of various parameters on nanofluid thermal conductivity. Journal of heat transfer 129(5),617-623

[10] Bhaskar Reddy N, Poornima T \& Sreenivasulu P (2016), Radiative heat transfer effect on MHD slip flow of Dissipating Nanofluid past an exponential stretching porous sheet. International of Journal of Pure and Applied Mathematics 109( 9) , 134 - 142

[11] Mei Zhang Jian Li (2009), Carbon nanotube in different shapes. Materials today, 12(6), 12-18.

[12] Xue Q Z (2005), Model for thermal conductivity of carbon nanotube-based composites. Physica B 368, 302-307.

[13] Hiroshi Ajiki \& Tsuneya Ando(1997), Carbon nanotubes as quantum wires on a cylinder surface. Solid State Communications 102(2-3), 135-142.

[14] Waqar A. Khan, Richard Culham \& Rizwan Ul Haq (2015), Heat Transfer Analysis of MHD Water Functionalized Carbon Nanotube
[4] Hayat T, Ijaz Khan M, Waqas M, Alsaedi A \& Farooq M (2017), Numerical simulation for melting heat transfer and radiation effects in stagnation point flow of carbon-water nanofluid. Computer Methods in Applied Mechanics and Engineering 315, 1011-1024.

[5] Irfan Rashid, Rizwan Ul Haq \& Qasem M Al-Mdallal (2017), Aligned magnetic field effects on water based metallic nanoparticles over a stretching sheet with PST and thermal radiation effects. Physica E: Low-dimensional Systems and Nanostructures 89, 33 42.

[6] Mahapatra TR \& Gupta AS (2002), Heat transfer in stagnationpoint flow towards a stretching sheet. Heat Mass Transfer $38,517-$ 521.

[7] Pop SR, Grosan T \& Pop I (2004), Radiation effects on the flow near the stagnation point of a stretching sheet. Tech. Mech, 25, $100-106$.

Flow over a Static/Moving Wedge. Journal of Nanomaterials 2015, Article ID 934367, 13 pages.

[15] Hayat T, Haider F, Muhammad T \& Alsaedi A (2017) Threedimensional rotating flow of carbon nanotubes with DarcyForchheimer porous medium. PLoS ONE 12(7), e0179576.

https://doi.org/10.1371/journal.pone.0179576

[16] Gireesha BJ, Mahanthesh B, Shivakumara IS, \& Eshwarappa KM (2016), Melting heat transfer in boundary layer stagnation-point flow of nanofluid toward a stretching sheet with induced magnetic field. Engineering Science and Technology, an International Journal 19, 313-321.

[17] Hayat T, Khursheed Muhammad, Farooq M \& Alsaedi A (2016), Melting heat transfer in stagnation point flow of carbon nanotubes towards variable thickness surface. AIP Advances 6, 015214

[18] Sunday Kolawole Adegbie, Olubode Kolade Ko Riko \& Isaac Lare Animasaun (2016), Melting heat transfer effects on stagnation point flow of micropolar fluid with variable dynamic viscosity and thermal conductivity at constant vortex viscosity. Journal of the Nigerian Mathematical Society 35, 34 - 47.

Table 3: The values of $-f^{\prime \prime}(0)$ and $-\theta^{\prime}(0)$ for different values of physical parameters.

\begin{tabular}{|c|c|c|c|c|c|c|c|c|c|c|c|c|}
\hline \multirow{2}{*}{$\phi$} & \multirow{2}{*}{$\gamma$} & \multirow{2}{*}{$A$} & \multirow{2}{*}{$\xi$} & \multirow{2}{*}{$R$} & \multirow{2}{*}{$\alpha$} & \multirow{2}{*}{$\beta$} & \multirow{2}{*}{$A^{*}$} & \multirow{2}{*}{$B^{*}$} & \multicolumn{2}{|c|}{$-f^{\prime \prime}(0)$} & \multicolumn{2}{|c|}{$-\theta^{\prime}(0)$} \\
\hline & & & & & & & & & SWCNTs & MWCNTs & SWCNTs & MWCNTs \\
\hline 0.1 & & & & & & & & & 1.07131 & 1.05822 & -0.54545 & -0.55944 \\
\hline 0.3 & & & & & & & & & 0.81879 & 0.78815 & -0.35062 & -0.35308 \\
\hline 0.5 & 0.1 & & & & & & & & 0.57394 & 0.538045 & -0.30023 & -0.29918 \\
\hline & 0.3 & & & & & & & & 0.83151 & 0.80681 & -0.46803 & -0.46639 \\
\hline & 0.5 & 0.1 & & & & & & & 0.84838 & 0.82839 & -0.57281 & -0.56807 \\
\hline & & 0.3 & & & & & & & 0.77474 & 0.75036 & -0.37528 & -0.36233 \\
\hline & & & $\pi / 4$ & & & & & & 0.73411 & 0.69769 & -0.36061 & -0.35712 \\
\hline & & & $\pi / 6$ & 0.1 & & & & & 0.81616 & 0.78549 & -0.35623 & -0.35919 \\
\hline & & & & 0.3 & & & & & 0.81752 & 0.78686 & -0.35339 & -0.35605 \\
\hline & & & & 0.5 & 0.1 & & & & 0.81879 & 0.78815 & -0.35062 & -0.35308 \\
\hline & & & & & 0.3 & & & & 0.68421 & 0.66524 & -0.34281 & -0.34463 \\
\hline & & & & & 0.5 & 0.1 & & & 0.59032 & 0.57771 & -0.33698 & -0.33819 \\
\hline & & & & & & 0.3 & & & 0.88577 & 0.82427 & -0.35806 & -0.36284 \\
\hline & & & & & & & 0.0 & & 0.83849 & 0.80411 & -0.30865 & -0.31687 \\
\hline & & & & & & & -0.1 & 0.1 & 0.84242 & 0.81208 & -0.30041 & -0.29908 \\
\hline & & & & & & & & 0.0 & 0.84534 & 0.80957 & -0.29418 & -0.30467 \\
\hline & & & & & & & & -0.1 & 0.85004 & 0.81887 & -0.28451 & -0.28405 \\
\hline
\end{tabular}

